\title{
HEMORRAGIA DIGESTIVA PROVOCADA POR TUMOR ESTROMAL GASTROINTESTINAL AVANÇADO DE DUODENO
}

\section{Gastrointestinal hemorrhage caused by advanced duodenal gastrointestinal stromal tumor}

\author{
Ruy Jorge CRUZ Jr, Nicolas GLYNIADAKIS, Rafael Noronha CAVALCANTE, \\ Lourdes Alves CEPEDA, Rodrigo VINCENZI
}

ABCDDV/576

Cruz Jr RJ, Glyniadakis N, Cavalcante RN, Cepeda LA, Vincenzi R. Hemorragia digestiva provocada por tumor estromal gastrointestinal avançado de duodeno. ABCD Arq Bras Cir Dig 2007;20(4):290-2

RESUMO - Introdução - O tumor estromal gastrointestinal (GIST) é neoplasia pouco freqüente, sendo rara a combinação de acometimento duodenal e hemorragia digestiva, por isso apresenta-se este relato. Relato do caso - Homem de 64 anos admitido com quadro de dor abdominal, melena e tumoração palpável em epigástrio e hipocôndrio esquerdo, sendo notado um tumor de paredes espessadas e conteúdo cístico na tomografia computadorizada de abdome, em topografia de cauda pancreática. Encontrado na laparotomia de urgência tumor em quarta porção duodenal com invasão de cólon em ângulo esplênico, sendo realizada ressecção em bloco do duodeno acometido, segmento de cólon transverso e descendente, com boa evolução pós-operatória. Diagnosticado por imunoistoquímica GIST de duodeno com invasão de parede colônica, sendo o tratamento complementado com mesilato de imatinib. Conclusão - A hemorragia digestiva é uma das possíveis complicações do GIST. Apenas o tratamento cirúrgico precoce é capaz de prevenir as graves complicações do choque hemorrágico.

DESCRITORES - Neoplasia gastrointestinal. Hemorragia gastrointestinal. Células estromais, duodeno.

\section{INTRODUÇÃO}

O tumor estromal gastrointestinal (GIST) apesar de ser o tipo de neoplasia mesenquimal mais comum do trato gastrointestinal, corresponde a apenas 3\% de todas as neoplasias malignas do sistema digestório. O recente avanço de técnicas de imunoistoquímica permitiu a diferenciação deste tipo de tumor em relação à outras neoplasias mesenquimais, mais especificamente leiomiomas e leiomiossarcomas, ocorrendo aumento no reconhecimento desta doença e conseqüentemente maior número de diagnósticos nos últimos anos. $\mathrm{O}$ acometimento duodenal é raro, sendo o estômago o órgão com maior incidência de GIST. Relata-se a seguir um caso de GIST avançado de duodeno complicado por hemorragia digestiva.

\section{RELATO DO CASO}

Paciente de 64 anos, sexo masculino, negro, admitido no serviço de emergência com quadro de dor abdominal e melena há um dia. Apresentava como co-morbidades hipertensão arterial sistêmica e episódio prévio de acidente vascular cerebral. Ao exame físico encontrava-se descorado, desnutrido, com freqüência cardíaca de 90 bpm e pressão arterial sistólica de

Trabalho realizado na Unidade de Emergência do Hospital Estadual do Grajaú Prof. Liberato John Alphonse Di Dio - Faculdade de Medicina da Universidade de Santo Amaro, São Paulo, SP.

Endereço para correspondência: Rodrigo Vincenzi, e-mail: rodvince@gmail.com
120 mmHg. À palpação abdominal apresentava tumoração endurecida e pouco móvel, de aproximadamente $10 \mathrm{~cm} \mathrm{x}$ $10 \mathrm{~cm}$, ocupando a região epigástrica e parte do hipocôndrio esquerdo. Exames laboratoriais na admissão incluíam nível de hemoglobina de $7,4 \mathrm{mg} / \mathrm{dL}$ e leucocitose de $12.800 / \mathrm{mm}^{3}$, não apresentando alterações nos testes de coagulação. Foi submetido à endoscopia digestiva alta, sendo notada a presença de sangue em segunda e terceira porções duodenais, não caracterizando-se a origem do sangramento. Realizada tomografia computadorizada de abdome, observou-se tumoração em topografia de cauda pancreática, com paredes espessadas e conteúdo de aspecto cístico em seu interior, sem calcificações, medindo aproximadamente $12 \mathrm{~cm}$ x $10 \mathrm{~cm}$ (Figura 1).

Vinte e quatro horas após sua admissão, o paciente apresentou aumento do sangramento e queda dos níveis de hemoglobina $(5,3 \mathrm{mg} / \mathrm{dL})$, apesar da transfusão de seis concentrados de hemácias. Optou-se então por laparotomia exploradora, identificado-se no intra-operatório grande tumoração proveniente de quarta porção duodenal $(10$ x 15 x $9 \mathrm{~cm}$ ), firmemente aderida em ângulo esplênico de cólon, sem evidências de doença metastática ou ascite à inspeção da cavidade (Figura 2). Realizada ressecção em bloco incluindo a $4^{\mathrm{a}}$ porção duodenal, segmento de jejuno proximal, cólon transverso distal e descendente proximal, com anastomose duodeno-jejunal primária e colostomia terminal (Figura 3). A evolução pós-operatória foi sem intercorrências, recebendo alta no décimo dia de pós-operatório. 


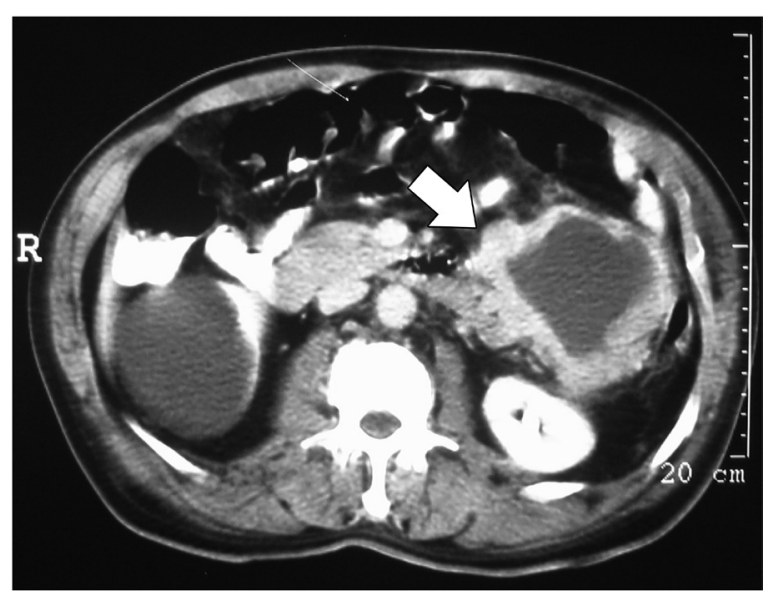

FIGURA 1 - Achado tomográfico de tumoração com paredes sólidas e conteúdo cístico em seu interior, indicada pela seta branca.

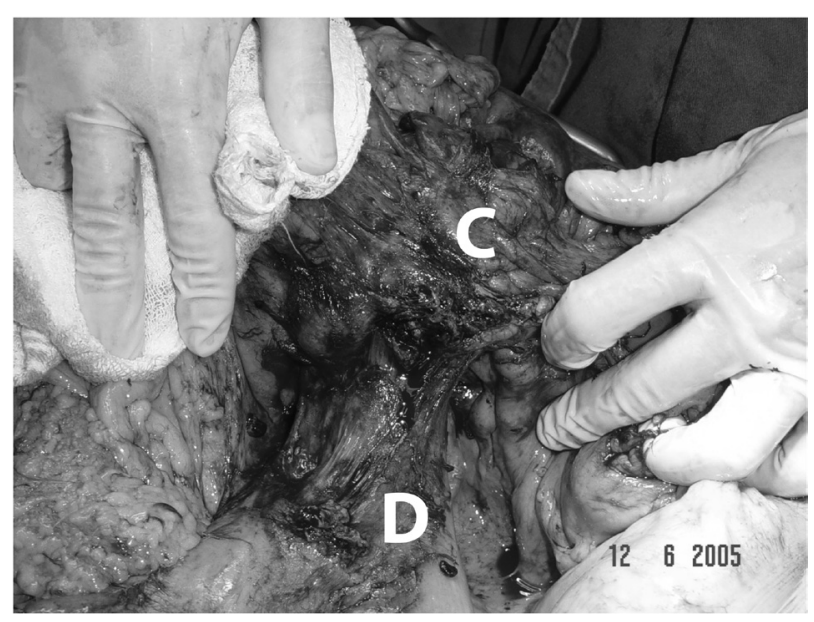

FIGURA 2 - Achado intra-operatório de GIST com origem em duodeno (D) e invasão de parede de cólon em ângulo esplênico $(\mathrm{C})$.

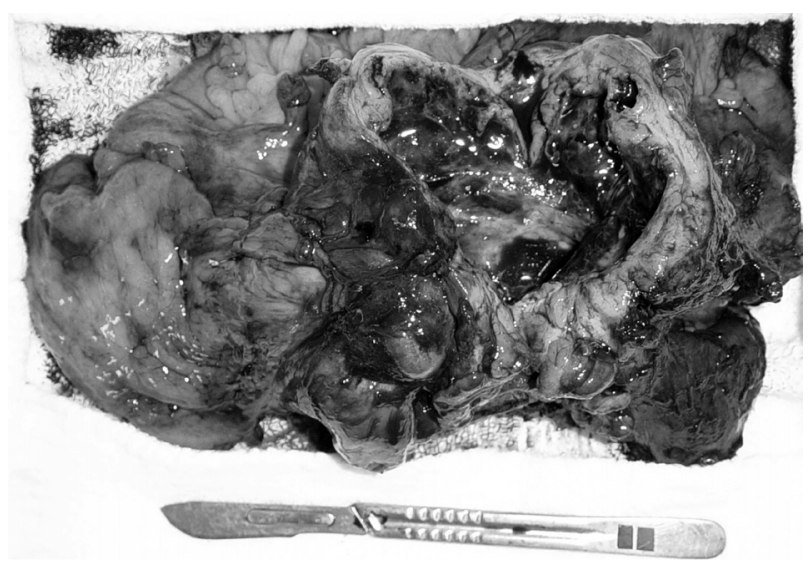

FIGURA 3 - Espécime cirúrgico em bloco de duodeno (D), cólon transverso (CT), cólon descendente e do GIST. A seta branca aponta o tumor e sua cavidade anteriormente preenchida por sangue, após a abertura do duodeno e do tumor.
Em acompanhamento ambulatorial, 12 meses após o tratamento cirúrgico, não apresentava sinais de recidiva da neoplasia, sendo que no pós-operatório o tratamento foi complementado com o uso do mesilato de imatinib, na dose de $400 \mathrm{mg}$ ao dia.

$\mathrm{O}$ exame anátomo-patológico da peça diagnosticou GIST de duodeno com invasão de serosa e camada muscular de cólon, com pouca proliferação celular, apresentando positividade para c-kit, desmina (D33), vimentina (V9) e proteína S-100 no exame imunohistoquímico.

\section{DISCUSSÃO}

A hemorragia digestiva é uma das apresentações freqüentemente encontradas em pacientes com GIST, seja de maneira crônica ou aguda. Tumorações abdominais palpáveis, dor abdominal e episódios de sub-oclusão intestinal completam a lista dos sintomas mais comuns neste tipo de neoplasia ${ }^{1}$. Entretanto, o diagnóstico etiológico do sangramento pode não ser evidente na avaliação diagnóstica inicial, como no caso aqui apresentado, já que o tumor encontrava-se na região mais distal do duodeno, não acessível ao endoscópio. Em outro caso operado por este mesmo grupo de autores, no qual o paciente apresentava um GIST gigante $(32$ × $22 \mathrm{~cm})$ de terço distal de estômago, a apresentação inicial foi semelhante (tumor palpável e hemorragia digestiva alta). Novamente, devido à discreta alteração encontrada na mucosa gástrica, que encontrava-se com uma pequena lesão ulcerada sem sangramento ativo, o diagnóstico não foi obtido por endoscopia.

Estes dois casos revelam uma das características marcantes destes tumores. O crescimento exofítico destas lesões, iniciado na camada submucosa do órgão, faz com que grandes tumores produzam pequenas alterações na camada mucosa, sendo mais comuns episódios de hemorragia digestiva por ruptura intra-luminal ao invés de sintomas de obstrução mecânica intrínseca, se considerar-se os tamanhos volumosos destas lesões ${ }^{2}$.

O tratamento cirúrgico do GIST, independentemente do sítio de origem, consiste em ressecção em bloco e de implantes metastáticos em casos selecionados ${ }^{1,3,4}$. A linfadenectomia não deve ser rotineiramente empregada, já que a disseminação linfonodal é pouco comum ${ }^{1,3,4}$. Em relação ao duodeno, diversas abordagens são descritas, dependendo da localização da neoplasia neste órgão, variando de ressecções segmentares, associadas ou não a derivações do conteúdo gástrico, a procedimentos mais complexos, como a gastroduodenopancreatectomia ${ }^{5,6}$.

O comportamento atípico desta neoplasia gera dificuldades no estabelecimento do diagnóstico de malignidade destes tumores. De maneira geral, 20 a 30\% dos GIST apresentam comportamento maligno ${ }^{1}$. Na ausência de lesões metastáticas visíveis, o tamanho e a atividade mitótica do tumor são os critérios normalmente empregados na diferenciação de malignidade ou não. Tumores com tamanho acima de $5 \mathrm{~cm}$ e com número de mitoses entre 5 a 10 em 50 campos de grande aumento 
possuem grande potencial maligno ${ }^{1,3,4,7,8}$.

Os índices elevados de recorrência desta doença após o tratamento exclusivamente cirúrgico foram responsáveis pelo desenvolvimento de novas modalidades de tratamento adjuvante, sendo atualmente o mesilato de imatinib o agente mais estudado. DeMatteo et $\mathrm{al}^{7}$ relatam índice de recidiva neoplásica de $40 \%$ em acompanhamento de dois anos, em um grupo de 80 pacientes submetidos à ressecção cirúrgica completa por GIST primário não metastático. Neste estudo, a sobrevida média dos pacientes foi de 66 meses para os casos de tumor primário com ressecção completa e 22 meses para os com tumores irressecáveis ou submetidos a ressecção incompleta ${ }^{7}$. Neste trabalho, entretanto, o tratamento adjuvante não fazia parte do protocolo, sendo avaliada praticamente apenas a resposta da doença ao tratamento cirúrgico isolado.
O uso do mesilato de imatinib vem sendo estudado em diferentes cenários clínicos. Estudos relatam seu uso em pacientes com doença metastática ou irressecável, com redução marcante do tamanho do tumor, ou ainda de maneira neoadjuvante ou adjuvante em pacientes com ressecções completas, porém com tumores com maiores chances de recidiva, devido ao seu tamanho ou atividade mitótica ${ }^{3,9}$. É esperado que novos estudos possam determinar com mais clareza as situações em que o tratamento com o imatinib seja mais eficaz.

\section{CONCLUSÃO}

Apesar da baixa freqüência desta neoplasia, a ocorrência de hemorragia digestiva neste grupo de pacientes não é incomum, sendo o tratamento cirúrgico precoce a única forma de se evitar as graves conseqüências do choque hemorrágico.

Cruz Jr RJ, Glyniadakis N, Cavalcante RN, Cepeda LA, Vincenzi R. Gastrointestinal hemorrhage caused by advanced duodenal gastrointestinal stromal tumor. ABCD Arq Bras Cir Dig 2007;20(4):263

ABSTRACT - Background - Gastrointestinal stromal tumor (GIST) represents an uncommon form of neoplasm. The combination of duodenal GIST and gastrointestinal bleeding consist of a rare presentation for such tumors. Aim - To report duodenal GIST case complicated by gastrointestinal bleeding. Case Report - A 64-year-old male was admitted presenting abdominal pain, melena and a palpable mass in epigastrium and left upper abdomem regions. CT scan reveled a thick wall tumor containing cystic content in the pancreatic tail topography. At emergency laparotomy, a tumor in the fourth portion of the duodenum presenting colonic invasion in splenic flexure was found. En-bloc resection of the tumor was carried out, included the fourth portion of the duodenum and the transverse and descending colon, without postoperative complications. Immunohistochemical staining of the resected specimen confirmed the diagnosis of duodenal GIST with colon wall invasion. Conclusion - Gastrointestinal hemorrhage is one of the possible complications of GIST. Early surgical treatment is the only effective therapeutic option to avoid severe complications of hemorrhagic shock.

HEADINGS - Gastrointestinal Neoplasm. Gastrointestinal hemorrhage. Stromal cells, duodenum.

\section{REFERÊNCIAS}

1. Nowain A, Bhakta H, Pais S, Kanel G, Verma S. Gastrointestinal stromal tumors: clinical profile, pathogenesis, treatment strategies and prognosis. J Gastroenterol Hepatol 2005; 20:818-824.

2. Mehta RM, Sudheer VO, John AK, Nandakumar RR, Dhar PS, Sudhindran S, Balakrishnan V. Spontaneous rupture of giant gastric stromal tumor into gastric lumen. World J Surg Oncol 2005; 3:11.

3. Wu PC, Langerman A, Ryan CW, Hart J, Swiger S, Posner MC. Surgical treatment of gastrointestinal stromal tumors in the imatinib (STI-571) era. Surgery 2003; 134:656-666.

4. Blay J-Y, Bonvalot S, Casali P, Choi H, Debiec-Richter M, Dei Tos AP, Emile J-F, Gronchi A, Hogendoorn PCW, Joensuu H, Le Cesne A, Mac Clure J, Maurel J, Nupponen N, Ray-Coquard I, Reichardt P, Sciot R, Stroobants S, van Glabbeke M, van Oosterom A, Demetri GD. Consensus meeting for the management of gastrointestinal stromal tumors. Ann Oncol 2005; 16:566-578.

5. Parreira JG, Freitas W, Rasslan S. Upper gastrointestinal hemorrhage due to duodenal stromal tumor. Arq Gastroenterol 2003; 40:188-191.
6. Sakamoto Y, Yamamoto J, Takahashi H, Kokudo N, Yamaguchi T, Muto T, Makuuchi M. Segmental resection of the third portion of the duodenum for a gastrointestinal stromal tumor: a case report. Jpn J Clin Oncol 2003; 33:364366.

7. DeMatteo RP, Lewis JJ, Leung D, Mudan SS, Woodruff JM, Brennan MF. Two hundred gastrointestinal stromal tumors. Recurrence patterns and prognostic factors for survival. Ann Surg 2000; 231:51-58.

8. Shinomura Y, Kinoshita K, Tsutsui S, Hirota S. Pathophysiology, diagnosis, and treatment of gastrointestinal stromal tumors. J Gastroenterol 2005; 40:775780.

9. Demetri GD, von Mehren M, Blanke CD, Van den Abbeele AD, Eisenberg B, Roberts PJ, Heinrich MC, Tuveson DA, Singer S, Janicek M, Fletcher JA, Silverman SG, Silberman SL, Capdeville R, Kiese B, Peng B, Dimitrijevic S, Druker BJ, Corless C, Fletcher CD, Joensuu H. Efficacy and safety of imatinib mesylate in advanced gastrointestinal stromal tumors. N Engl J Med 2002; 347:472-480.

Conflito de interesse: não há

Fonte financiadora: não há

Recebido para publicação em: 04/05/2007 Aceito para publicação em: 10/09/2007 